\title{
The Mechanisms of Adverse Reactions to Oseltamivir: Part II. Delayed Type Reactions
}

\section{Rokuro Hama*}

Japan Institute of Pharmacovigilance, Non-profit Organization, Osaka, Japan

*Corresponding author: Rokuro Hama, Japan Institute of Pharmacovigilance, Non-profit Organization, Osaka, Japan, Tel: +81-6-6771-6314; Fax: +81-6-6771-6347; Email: ec00724@nifty.com

Received date: October 16, 2015; Accepted date: November 21, 2015; Published date: November 28, 2015

Copyright: @ 2015 Hama R. This is an open-access article distributed under the terms of the Creative Commons Attribution License, which permits unrestricted use, distribution, and reproduction in any medium, provided the original author and source are credited.

\begin{abstract}
Oseltamivir is recommended for treatment and prophylaxis of Influenza in persons at higher risk for Influenza complications such as individuals with diabetes, neuropsychiatric illnesses, and respiratory, cardiac, renal, hepatic or hematological diseases. However, a recent systematic review reported that reduction of antibody production, renal disorders, hyperglycemia, psychiatric disorders, and QT prolongation may be related to Oseltamivir use. The underlying mechanisms of these effects are reviewed.

There is decisive evidence that administration of a clinically compatible dose of Oseltamivir in mice challenged by a respiratory syncytial virus (RSV) that lacks a neuraminidase gene showed symptom-relieving effects and inhibition of viral clearance. These effects were accompanied by decreased level of $\mathrm{T}$ cell surface sialoglycosphingolipid (ganglioside) GM1 that is regulated by the endogenous neuraminidase in response to viral challenge. Clinical and non-clinical evidence supports the view that the usual dose of Oseltamivir suppresses pro-inflammatory cytokines such as interferon-gamma, interleukin-6, and tumor necrosis factor-alpha almost completely in experimentally infected Influenza viruses in humans with partial suppression of viral shedding.
\end{abstract}

Animal toxicity tests support the clinical evidence with regard to renal and cardiac disorders (bradycardia and QT prolongation) and do not disprove the metabolic effect. Reduction of antibody production and cytokine induction and renal, metabolic, cardiac, and prolonged psychiatric disorders after Oseltamivir use may be related to inhibition of the host's endogenous neuraminidase. While the usual clinical dose of Zanamivir may not have this effect, a higher dose or prolonged administration of Zanamivir and other neuraminidase inhibitors may induce similar delayed reactions, including reduction of antibody and/or cytokine production.

Keywords: Antibody; Endogenous neuraminidase; Neuraminidase inhibitor; Neuropsychiatric adverse effects; Oseltamivir; Proinflammatory cytokines; QT prolongation; Renal impairment

\section{Introduction}

Neuraminidase inhibitors (NIs) are expected to reduce complications of Influenza, especially in persons at higher risk for Influenza complications [1,2], and Oseltamivir is included in the Model List of Essential Medicines [3]. Persons at higher risk include those with diabetes, neuropsychiatric illnesses, and respiratory, cardiac, renal, hepatic, or hematological diseases [1,2]. However, serious neuropsychiatric adverse reactions to Oseltamivir, including sudden deaths and abnormal behaviours leading to accidental death, have been reported since the drug was introduced into medicine [4-8]. In Japan, Oseltamivir has been contraindicated in principle for children and adolescents aged 10 to 19 years since 2007 due to concern about the risk of abnormal behaviours [4-7]. Adverse reactions to Oseltamivir include sudden and delayed onset types and others [6,7]. Sudden death is another concern about Oseltamivir use [5-8].

The Cochrane's neuraminidase inhibitors team showed in its latest review [9] that neuraminidase inhibitors (NIs), including Oseltamivir and Zanamivir, have symptom-relieving effects in adults, but it is unclear whether the same effects occur in children. Oseltamivir reduced a four-fold increase in antibody titre $[9,10]$. Evidence of reduction in hospitalization was not shown for either NI. Oseltamivir did not reduce complications, including pneumonia classified as serious or which led to study withdrawal. Both NIs reduced "laboratory confirmed symptomatic Influenza infection," but not Influenza-like illness. Oseltamivir increased risk of nausea, vomiting, headache, psychiatric, renal, and diabetic/hyperglycemic events, and pain in limbs, but Zanamivir did not. Oseltamivir reduced diarrhea and apparent cardiac events, but induced prolonged QTc time (Bazett correction) [9].

This review discusses the biological base of adverse reactions, in particular those of the delayed type.

\section{Brief Description of Neuraminidase Inhibitors}

\section{Neuraminidase inhibitors and medical use}

Neuraminidase inhibitors (NIs) comprise inhaled Zanamivir (Relenza, GlaxoSmithKline), oral Oseltamivir (Tamiflu, Gilead Sciences, F. Hoffman-La Roche and Chugai in Japan), parenteral Peramivir [11] (BioCryst Ltd and Shionogi), inhaled laninamivir (Daiichi Sankyo Co. Ltd) [12], and others still under development [13]. Zanamivir and Oseltamivir have been marketed in the US since 1999, and subsequently in other countries including Japan. Peramivir and 
Laninamivir were put on the market in Japan in January and September 2010, respectively.

F. Hoffman-La Roche (Roche) donated Oseltamivir to the World Health Organization (WHO), and the WHO decided to recommend stockpiling neuraminidase inhibitors (especially Oseltamivir) for use in the event of a pandemic [14]. Prior to the emergence of Influenza A/ H1N1 in 2009, governments worldwide stockpiled 220 million treatments of Oseltamivir, swelling sales since the start of 2003 to CHF 7.6 billion [15]. The use of NIs has increased dramatically since the outbreak of A/H1N1 in April 2009.

Between the first approval in the US and the 2006/2007 season, approximately 48 million patients received a prescription for Oseltamivir worldwide [16], while 4 million patients received a prescription for Zanamivir [17]. Japan, the US, and the other countries consumed $76 \%, 21 \%$, and $3 \%$ of the world total of Oseltamivir, respectively [16]. For children (suspension), $85 \%$ was consumed in Japan [17].

Zanamivir was rarely prescribed (less than $1 \%$ of total antiviral prescriptions for Influenza) in Japan before the Japanese Ministry of Health, Labour and Welfare (MHLW) contraindicated Oseltamivir use in patients aged 10 years to 19 years in March 2007 [4,6]. After this restriction, prescriptions of Oseltamivir decreased substantially. After laninamivir was put on the market in Japan in September 2010, its share increased rapidly. In the 2013/14 season, the prescription shares of Oseltamivir, Zanamivir, laninamivir, and peramivir were $36 \%, 19 \%$, $42 \%$, and $3 \%$ in Japan, respectively [18].

\section{Mode of Action and Pharmacokinetics in Non- Infectious State}

Although NIs may reduce the ability of the virus to penetrate the mucus in the very early stage of infection [19-24], their main mechanism of action is thought to lie in their ability to interfere with the release and spread of progeny Influenza virus from infected host cells by inhibiting neuraminidase of Influenza viruses [21,23,24]. It is generally believed that Oseltamivir most likely reduces symptom duration by reducing viral load, and via the spread and release of cytokines [25]. However, the full prescription information of Oseltamivir (revised in April 2010) states "The concentrations of Oseltamivir carboxylate required for inhibition of Influenza virus in cell culture were highly variable depending on the assay method used and the virus tested. The relationship between the antiviral activity in cell culture, inhibitory activity in the neuraminidase assay, and the inhibition of Influenza virus replication in humans has not been established" [24]. It is not clearly stated that the mechanisms of symptom relief are derived from reduction of viral load.

Oseltamivir phosphate (OP) is an ethyl ester prodrug that requires ester hydrolysis for its conversion to the active form of the neuraminidase inhibitor, Oseltamivir carboxylate (OC). The brand name drug of OP capsule (Tamiflu capsule) contains $75 \mathrm{mg}$ of Oseltamivir expressed as free base (OT), which is compatible with 98.6 $\mathrm{mg}$ of OP. OP dissociates in the gastrointestinal tract to form OT, which is absorbed and metabolised into OC by hepatic carboxylesterase (hCE).

In healthy volunteers, the area under the curve (AUC) of OP is $3 \%$ to $5 \%$ that of OC. The penetration of OP across the blood-brain barrier (BBB) is restricted (less than 10\%) by P-glycoprotein (P-gp) in mature and non-infected animals [26-28].
When healthy adult volunteers were administered with $75 \mathrm{mg}$ of OT (equivalent to $98.6 \mathrm{mg}$ of $\mathrm{OP}$ ), approximate average $\mathrm{C}_{\max }(\mathrm{ng} / \mathrm{ml}), \mathrm{T}_{\max }$ (h), AUC (ng.h/ml), and elimination half-life (t1/2:hs) of OP were as follows, respectively: $60 \mathrm{ng} / \mathrm{ml}, 0.7-2 \mathrm{hs}, 150-200 \mathrm{ng} . \mathrm{h} / \mathrm{ml}$, and 1.2 h-1.9 h. For OC, they were $200 \mathrm{ng} / \mathrm{ml}-300 \mathrm{ng} / \mathrm{ml}, 4-5 \mathrm{~h}, 3000 \mathrm{ng} \mathrm{h} /$ $\mathrm{ml}-4000 \mathrm{ng} \mathrm{h} / \mathrm{ml}$, and $5 \mathrm{~h}-10 \mathrm{~h}$ [29]. The pharmacokinetic (PK) parameters in healthy children aged 3 years or older are not very different from those in adults [29]. In the 5 patients with decreased creatinine clearance $(<30 \mathrm{ml} / \mathrm{min})$, after 6 days administration of 100 $\mathrm{mg}$ of OT (as free form), PK parameters ( \pm SD) of OC were as follows: Cmax: $4052( \pm 1519)$ ng/ml, Tmax: 5.20 ( \pm 1.11$)$, AUC0-12:43086 ng $\mathrm{h} / \mathrm{ml}( \pm 18068)$, and t1/2:16.1 ( \pm 2.69$)[29]$.

In the 12 patients who were hemo-dialyzed, PK parameters $( \pm \mathrm{SD})$ after $75 \mathrm{mg}$ of OT were as follows: Cmax: 2131 ( \pm 533) ng/ml, Tmax: 27.3 ( \pm 6.9), AUC0-last: $106314( \pm 26.029) \mathrm{ng} \mathrm{h} / \mathrm{ml}$, and $\mathrm{t} 1 / 2: 159$ (these data were calculated before the next dialysis, 48 hours after the previous dialysis; SD was not given) [29]. Thus, almost all OC is secreted in urine, and dose adjustment is necessary if the patients' creatinine clearance is less than $30 \mathrm{ml} / \mathrm{min}[24,29,30]$.

\section{Evidence on Adverse Reactions to Oseltamivir in Humans and in Other Animals}

To date, it is postulated that adverse effects of Oseltamivir include sudden onset type, delayed onset type, and others [6,7]. This chapter mainly summarizes the delayed onset type adverse reactions to Oseltamivir reported in the clinical trials and epidemiological studies, which may help to explain possible adverse effects of other neuraminidase inhibitors. A very brief review of the sudden onset type neuropsychiatric adverse effects of Oseltamivir is also provided.

\section{Sudden onset neuropsychiatric reactions}

The sudden onset type reactions include nausea, vomiting, and hypothermia, as well as neuropsychiatric reactions such as abnormal behaviours, hallucination, and sudden respiratory arrest followed by cardiac arrest and death [6,7,31]. These appear very shortly (from less than 1 hour to 12 hours at most) after the first dose of Oseltamivir, and disappear rapidly unless they induce respiratory arrest and sudden death, or sequelae $[6,7,31]$. They may disappear even if Oseltamivir is continuously taken, although symptoms may reappear if the drug is taken several times. The underlying mechanisms of sudden-onset type reactions are discussed elswhere $[6,31]$.

\section{Delayed onset reactions with prolonged time for recovery}

Delayed onset reactions include disorders of various organs and systems such as renal, metabolic, cardiac, hepatic, hematological, immune, nervous, psychiatric, and general systems (fatigue or malaise). Most of the reactions of this type appear at least a few days after commencement of Oseltamivir intake, although QTc prolongation may be closely related to the plasma concentration of Oseltamivir carboxylate, even at first dose (details described below). Duration of symptoms from these reactions, especially neuropsychiatric ones, tends to be prolonged: for months or even years [6].

\section{Inhibition of antibody production and reinfection}

Oseltamivir significantly reduced the odds of patients having a 4fold antibody rise, by almost $20 \%$ (risk ratio by $8 \%$ ), according to a 
meta-analysis of 8 reports (10 studies) $[9,10]$. Heterogeneity was not significant $\left(\mathrm{I}^{2}=4 \%\right)$.

Attenuation of secretory $\operatorname{IgA}(\operatorname{sg} \mathrm{A})$ was more marked [32-35]. Sawabuchi et al. reported that lower induction of sIgA against the Influenza A virus was observed in children treated with Oseltamivir in comparison with children treated without Oseltamivir. The odds of a child's sIgA level increasing more than 5 -fold were significantly lower in children treated without Oseltamivir: OR=0.11 (95\% CI: 0.02, 0.61, $\mathrm{p}=0.008$ ) [32]. Anti-Influenza A virus sIgA attenuation was observed in children treated both with Oseltamivir or with Zanamivir [33].

These findings are consistent with evidence from animal tests using sub-clinical doses of Oseltamivir in Influenza A/H1N1 infected mice [34,35]. Non-significant slight reduction of hemagglutinin (HA) specific IgG antibody in the serum and spleen was reported, while HA specific secretory IgA antibody (s-IgA $\mathrm{Ab}$ ) in nasal wash and bronchoalveolar fluids (BALF) was significantly reduced: by approximately $80 \%$ on day 12 [34].

In human clinical trials, Zanamivir at the usual dosage did not reduce antibody (anti-HA $\mathrm{Ab}$ ) production [9], but attenuated $\operatorname{sIgA}$ antibody significantly [33]. In a double blind, placebo controlled trial with healthy volunteers designed to investigate the effect of Zanamivir treatment $(20 \mathrm{mg} /$ day for 14 days $)$ on the humoral immune response to Influenza, the Zanamivir group responded with significantly lower antibody titres to the H1N1 [36]. Levels of pro-inflammatory cytokines including IL- 6 , TNF- $\alpha$, IFN- $\gamma$, and other chemokines were almost completely suppressed in the viral challenge RCT using a very high dose $(600 \mathrm{mg})$ of intravenous Zanamivir before inoculation of Influenza virus in human adults [37].

Shinahara et al. [33] reported: "Even under the spread of a new virus subtype in $2009 / 2010$, only $8.6 \%$ of the children of the no-treatment group were re-infected. However, the proportions of children treated the previous year with Oseltamivir and Zanamivir who developed reinfection in 2009-2010 were significantly higher at $37.3 \%$ and $45.0 \%$, respectively $(\mathrm{P}<0.01)$, than those of the no-treatment group."

Several cases of re-infection with the same Influenza virus within one season were reported [38,39]. Kopel et al. [38] reported a 13-yearold boy with cerebral palsy who 3 times had episodes of fevering with positive 2009A/H1N1 Influenza detected by the RT-PCR method. He was treated with the standard dose ( $75 \mathrm{mg}$ b.i.d for 5 days) of Oseltamivir at the time of the first episode. A second course of Oseltamivir was administered for 10 days with the dosage adjusted for age and doubled from that of the previous regimen. His HI titres were high, but the level of secretory IgA was not determined.

Renal impairment: Dose-related histological change in the renal tubules, increased urine volume, and increased relative weights of the liver and kidneys were observed at various doses in various animals treated for various periods [29].

For example, in the 6-month rat toxicity test, renal weights (both relative and absolute) increased, and histological examination revealed degenerating and regenerating changes in the renal tubular epithelia, basement membranes, and Bowman capsules; vacuolization in the renal tubular epithelia; and mineralization in the highest dose group $(761 \mathrm{mg} / \mathrm{kg})$. These histopathological findings were not reversed at 8 weeks after cessation of Oseltamivir intake. In the medium-dose group (152 mg/kg: HED $^{*} \mathrm{a}=24.5 \mathrm{mg} / \mathrm{kg}$ ), renal relative and absolute weights increased, and vacuolization in the renal tubular epithelia was observed [29]. NOAEL for renal impairment was $38 \mathrm{mg} / \mathrm{kg}$. HED for
$38 \mathrm{mg} / \mathrm{kg}$ is $6.1 \mathrm{mg} / \mathrm{kg}$ converted by body surface area and $5.5 \mathrm{mg} / \mathrm{kg}$ converted by AUC level of OC. These are 2.4 and 2.2 times higher than the usual dose for a person weighing $60 \mathrm{~kg}$. Renal impairment in the highest-dose group was accompanied by increased water intake, increased leukocyte count, and increased bilirubin, BUN, creatinine, urine volume, and NAG/creatinine ratio.

*a: HED (human equivalent dose) [40]. According to the "Guidance for Industry," for a person weighing $60 \mathrm{~kg}$, conversion factors for HED for mouse, rat, ferret, marmoset monkey, rabbit, and dog are 12.3, 6.2, $5.3,6.2,3.1$, and 1.8 , respectively.

Metabolic disorders: hyperglycemia and diabetes: Increased glucose level in the highest dose group $(1522 \mathrm{mg} / \mathrm{kg})$ was observed in a rat oral 2 -week test. The data were not described, but this increased glucose level was accompanied by increased leukocyte count, dose-related significant increase of BUN ( $<<0.01)$, increased weights of the kidney and the liver, mineralization of tubules of renal medulla (male: $8 / 10$ ), and mild-to-moderate accumulation of lung alveolar macrophages [29].

Cardiac disorders: bradycardia and QT prolongation: Oseltamivir decreased heart rate in the 9-month repeated toxicity test using marmoset monkeys. The average heart rate during treatment period with Oseltamivir was 328 beats/min, which was an $11 \%$ and $16 \%$ decrease compared with the control group (368 beat/min) and the average of baseline and recovery phase (392 beat $/ \mathrm{min}$ ), respectively [29].

In an experiment using beagle dogs to test the effects on cardiac functions such as QT time [41], mean baseline QTc intervals (msec \pm SE) were $417 \pm 16$ in the control (vehicle) group ( $n=4)$ and $374 \pm 2$ in the Oseltamivir carboxylate (OC) group $(n=4)$. This difference was significant $(\mathrm{p}=0.0372)$ according to the summary data $\mathrm{t}$-test. Other evidence suggested that variation was higher in the control group $(\mathrm{p}=0.005$; Bartlett's test) [42]. The average \pm standard error of QTc interval of anesthetized dogs intravenously infused with $100 \mathrm{mg} / \mathrm{kg}$ OC over 30 minutes significantly increased $(390 \pm 4)$ compared with that before infusion $(376 \pm 2)$. The average QTc recovered $(374 \pm 6)$ at 1.5 hours after discontinuation, but the standard error became larger. QTc prolongation is closely related to the serum concentration of OC $[29,41,42]$. The reasons for such large and systemic imbalances are not known, but they are unlikely to have occurred by chance.

Bradycardia and QT prolongation that occurred in the animal tests were also observed in the randomized controlled trials in humans, and the occurrence was closely related to the timing of the increase in the concentration of Oseltamivir carboxylate [9].

Delayed type psychiatric symptoms: The author reported the following case of psychiatric reaction [6]. A 15-year-old junior high school boy with a body temperature of $39.2 \mathrm{C}$ due to Influenza B diagnosed by rapid testing took Tamiflu $75 \mathrm{mg}$ b.i.d. for 5 days. His body temperature was normalized on day 5 , but he felt lethargic. After he took the last $\left(10^{\text {th }}\right)$ dose of Tamiflu in the morning on day 6 , he went to school, sat on his desk, and began to sing loudly during a lesson. He could not communicate with his classmates. He seemed to be delirious. 4 days after this episode, his parents took him to a general hospital. Before admission, he commented, "There are insects on my mask," a sign of visual hallucinations. After admission, he tried to pull out his venous lines and attempted to go home, shouting "This is not a hospital but a nursing home for elderly people." He could not wait until his turn for examination and rushed out of the hospital into the street, where he narrowly avoided being run over by a car. On day 16 he was 
discharged, and on days 19 and 20 he came to know that he had behaved abnormally from records on his mobile phone. It took 13 days after the beginning of abnormal behaviour for his psychiatric symptoms to be completely resolved [6].

Among the four study reports of prophylaxis RCTs analysed in the systematic review [9], the case of longest recovery time was Subject $23639 / 3122$ in trial WV15825 [43]. The narrative description on module 1 of the clinical study report stated as follows:

"This 69-year-old female was hospitalized on study day 8 because of paranoid schizophrenia. Her medical history included paranoid schizoaffective disorder, hypertension, and coronary artery disease. Her medications included Ketoconazole, Amlodipine, Haloperidol and Lorazepam. On study day 8 she ran away from her place of residence, but she was found and transferred to hospital for medical treatment. Study medication was discontinued on study day 8. Paranoid schizophrenia of severe intensity was diagnosed. She subsequently absconded from the hospital and was found on study day 15 with moderate concussion. She was again hospitalized, for 10 days. The paranoid schizophrenia resolved within 68 days and was considered unrelated to study medication.

In the prophylaxis RCTs, 11 and 2 cases of psychiatric events with late onset and prolonged recovery (14 days and longer) were reported in the Oseltamivir and placebo groups, respectively. The pooled odds ratio was 3.37 (95\% CI: 1.11-10.23, $\mathrm{p}=0.032, \mathrm{I}^{2}=0 \%$ ).

No animal study was performed to confirm the association of psychotic reactions of delayed onset with prolonged recovery.

\begin{tabular}{|c|c|c|}
\hline System & Symptom group & Symptoms and reactions \\
\hline \multicolumn{3}{|c|}{ Sudden onset type reactions (by Oseltasmivir) } \\
\hline Digestive & Gastrointestinal & nausea, vomiting \\
\hline \multirow{3}{*}{ Central nervous system } & $\begin{array}{l}\text { Mild to moderate } \\
\text { symptoms }\end{array}$ & hypothermia, sleep, headache, vomiting, vulging of fontanelle \\
\hline & Psychiatric symptoms & $\begin{array}{l}\text { sensory disturbances, impairment of cognition, abnormal behaviour (suppressed or } \\
\text { excitatory), unconsciousness, paranoia, delusion, hallucination, psychosis, depression, } \\
\text { aggression, agitation, delirium, suisaidal (ideation, attempt, complete) }\end{array}$ \\
\hline & Respiratory suppression & $\begin{array}{l}\text { cyanosis, difficulty of breathing, hyperpnea, hypopnea, irregular breath, respiratory failure, } \\
\text { respiratory arrest, cardiorespiratory arrest, and death }\end{array}$ \\
\hline \multicolumn{3}{|c|}{ Delayed onset and/or prolonged type reactions } \\
\hline \multirow{4}{*}{ Immune/inflammatory/infectious } & antibody & reduction of antibody production, especially secretory $\lg \mathrm{A}$ at respiratory tract \\
\hline & cytokine & attenuated induction of cytokine and chemokines (IL-6, IFN- - , TNF- $\alpha$ etc) \\
\hline & cell & reduction of inflammatory cells in nasal wash and lung, reduction of $\mathrm{CD}^{+} \mathrm{T}$ cell in lung \\
\hline & reinfection etc & reinfection of influenza, pneumonia and exacerbation of other infections \\
\hline Renal & histology/function & $\begin{array}{l}\text { degenerating and regenerating changes in the renal tubular epithelia and Bowman capsules, } \\
\text { increased urine volume and kidney weight, proteinuria etc }\end{array}$ \\
\hline Metabolic & diabetic & hyperglycemia, exacerbation of diabetes mellitus, new onset diabetes \\
\hline Cardiac & contraction & decreased heart rate, bradycardia, QT prolongation \\
\hline Neurological & nociceptor & pain in limbs or other parts of the body \\
\hline Psychiatric & $\begin{array}{l}\text { delayed onset/ } \\
\text { prolonged type }\end{array}$ & $\begin{array}{c}\text { delayed onset psychiatric symtoms (abnormal behaviour, psycosis hallucination, delusion, } \\
\text { agitation, schizophrenic reactions, depression etc) }\end{array}$ \\
\hline Digestive & GI tract & bleeding \\
\hline Others & & bleeding (hepatic and/or hematological impairment) \\
\hline
\end{tabular}

Table 1: Spectrum of adverse reactions to neuraminidase inhibitors.

Other adverse effects (pneumonia, wheezing, gastric bleeding, and others): 3 of 6 rats treated with intravenous OC (at 12 times higher level than clinical area under the curve) for 2 weeks developed acute alveolitis [29]. Of the 3, 1 exhibited wheezing on day 14 and was sacrificed the next day. Diffuse hemorrhagic alveolitis (pneumonia) and pulmonary microvascular thromboembolism were observed in this animal. The safe level of intravenous OC dose is lower than twice the AUC of the usual clinical human dose.
In the marmoset monkey 7-day oral toxicity studies, all 4 animals treated with a 127-times-higher-than-HED dose of OT were sacrificed within 4 days ( 1 on day 2 and 3 on day 4 ) because they were near death after severe vomiting, sleep, hypoactivity, and collapse. Macroscopic reddening of the stomach mucosa and histologically mucosal bleeding with erosions, ulcers, and atrophy were observed in the stomachs of all animals.

The safety index (animal $\mathrm{AUC}_{0-24}$ with no toxicity by human average $\mathrm{AUC}_{0-24}$ when taking $75 \mathrm{mg}$ capsule b.i.d.) is 3 for the 4 -week 
toxicity studies in rats, 3 for the 6-month oral toxicity studies in rats, 8 for the 2-week oral toxicity study in rats, and 10 for the marmoset monkey 7-day oral toxicity study.

Potential adverse reactions to Oseltamivir and to other neuraminidase inhibitors: Potential adverse reactions to Oseltamivir and to the other neuraminidase inhibitors are summarised in Table 1.

\section{Mechanisms for Symptom Relief and the Host's Endogenous Neuraminidase}

Mechanisms for symptom relief are mainly discussed in this section, and those for delayed reactions are mainly discussed in the next section. Both mechanisms seem to be related to inhibition of the host's neuraminidase.

\section{Inhibition of the host's neuraminidase and symptom relief}

Symptom relief in RSV-infected mice by Oseltamivir: Decisive evidence is shown by Moore et al. [44], who reported that administration of a clinically compatible dose of Oseltamivir in mice challenged by a respiratory syncytial virus (RSV) that lacks a neuraminidase gene showed symptom-relieving effects (decreased weight loss) and inhibition of viral clearance. These effects were accompanied by decreased level of $\mathrm{CD}^{+} \mathrm{T}$ cell surface sialoglycosphingolipid (ganglioside) GM1, which is regulated by the endogenous sialidase/neuraminidase in response to viral challenge, along with suppression of cytokines expression. To date, no such study has been conducted for Zanamivir, Laninamivir, or Peramivir.

Decreased GM1 ganglioside and suppression of pro-inflammatory cytokines: In the human phase II randomized controlled trial with experimental infection [25], pro-inflammatory cytokines including IL-6, TNF- $\alpha$, and IFN- $\gamma$ were completely suppressed by Oseltamivir administered 28 hours after the experimental inoculation of the Influenza virus, while reduction of viral titre in nasal lavages was partial.

Attenuating induction of pro-inflammatory cytokines including IL- 6 , TNF- $\alpha$, and IFN- $\gamma$ is related to decreased secretion by immune cells including dendritic cells [45], by polymorphonuclear leukocytes [46], and by $\mathrm{CD}^{+}$T-cells $[44,47]$. Reduced cytokine induction is derived from decreased expression of GM1 ganglioside in these cells related to inhibition of the host's endogenous neuraminidase (or Sialidase) [44-47], especially Neu 3 (the third sub-type of neuraminidase mainly expressed in plasma membrane) [45].

Decreased GM1 ganglioside and pain: Crain et al. [48] reported that Oseltamivir at 100 to 1000 times lower HED than the clinical dose may affect the host's neuraminidase and reduce endogenous GM1 ganglioside, leading to some reactions in the host. They suggest that "Clinical administration of Oseltamivir at doses that result in inhibition of Influenza may also have an additional effect by decreasing GM1 levels in nociceptive neurons" [48].

\section{Animal infection model: symptoms, inflammatory, cytokine response, and viral load}

Ferret model: reduction of febrile, inflammatory response with little viral load change: The ferret model is one of the best animal models for human Influenza infection. Roche used this model and reported as follows in the protocol (Module II) of most clinical study reports for treatment randomized controlled trials $\left[43^{\star} \mathrm{a}\right]$ :
"Adult ferrets (4 per group) were inoculated with a virulent Influenza strain. Ro-0796 was administered orally at a dose of either 5 $\mathrm{mg} / \mathrm{kg}$ or $25 \mathrm{mg} / \mathrm{kg}$ b.i.d. for 3 days starting 2 hours post exposure. A control group of four ferrets received vehicle alone. In this experiment, Ro 64-0796 was shown to reduce the febrile response and reduce the number of inflammatory cells in nasal washing in a dose dependent manner. However, neither dose was demonstrated to reduce the viral titres obtained from the lungs or nasal washings of infected animals." Ro-0796 refers to Oseltamivir phosphate.

According to the published paper of Oseltamivir [49], the area under the curve (AUC) of the viral load of both $5 \mathrm{mg} / \mathrm{kg} /$ day and of 25 $\mathrm{mg} / \mathrm{kg} /$ day oral doses and peak viral load in the $5 \mathrm{mg} / \mathrm{kg} /$ day group were not reduced significantly, although average peak viral titres of the $25 \mathrm{mg} / \mathrm{kg}$ /day group was reduced significantly.

In contrast, AUC of the febrile response was reduced significantly in both Oseltamivir dose groups, dose-dependently. In addition, the total number of inflammatory cells in nasal wash obtained from infected animals in both Oseltamivir dose groups was continuously reduced for more than 96 hours.

The conversion factor for ferret dose to human equivalent dose (HED) for a person weighing $60 \mathrm{~kg}$ in the "Guidance for Industry" [40] is 5.3 . Therefore, $5 \mathrm{mg} / \mathrm{kg}$ and $25 \mathrm{mg} / \mathrm{kg}$ for adult ferrets correspond to approximately $1 \mathrm{mg} / \mathrm{kg}$ and $5 \mathrm{mg} / \mathrm{kg}$ for humans. These are almost equivalent doses and 4 to 5 times higher than the human single dose of Oseltamivir (75 mg/60 kg).

Hence, the clinical dose of Oseltamivir may reduce febrile response and the number of inflammatory cells in a nasal washing without significant reduction of viral titres obtained from the lungs or nasal washings of infected animals.

*a: Module II of the most CSRs for adult treatment RCTs including 2 pivotal RCTs (WV15670, WV15671) and others (WV15673/15697, WV15707, WV15708, WV15730, WV15758, WV15759/15871, WV15799, WV15812/872, WV15819/15876/15978, WV15825), except 3 CSRs (M76001, WV16277 and ML16369). These full CSRs are available in ref [43] http://datadryad.org/resource/doi:10.5061/dryad. 77471.

Mouse model: mild Influenza and lack of evidence of reduction of viral load: Oral administration of $10 \mathrm{mg} / \mathrm{kg}$ of OP per day caused a 100 -fold reduction in lung homogenate viral titres in mice infected with a $90 \%$ lethal dose of some strains of Influenza A or B viruses, and enhanced survival $[29,49]$. Similar experiments were reported for Peramivir [50-52].

However, in a study by Wong et al. [53] using mice infected with mild Influenza (inoculated with a non-lethal dose of Influenza virus), which is a better model for testing the effects of Oseltamivir in human seasonal Influenza, a clinically compatible dose of Oseltamivir $(10 \mathrm{mg} /$ $\mathrm{kg}=$ approximately $0.8 \mathrm{mg} / \mathrm{kg}$ as HED) administered (in 3 different experiments) at 4 hours before inoculation, 24 hours after inoculation, or 48 hours after inoculation showed no significant effect on viral titres at day 5 post-inoculation.

Wong et al. [53] observed that Oseltamivir markedly and significantly reduced lung inflammatory cell response and induction of pro-inflammatory cytokines and chemokines such as TNF- $\alpha$, IL- $1 \beta$, IL-6, granulocyte-macrophage colony-stimulating factor (GM-CSF), keratinocyte-derived chemokine (KC), Macrophage inflammatory protein-1 $\alpha$ (MIP-1 $\alpha$ ), and Monocyte chemotactic protein-1 (MCP-1) whether administered prophylactically or therapeutically. However, 
Page 6 of 10

these were accompanied by small non-significant effects on viral titres. Based on these findings, the researchers discussed the possibility of intrinsic anti-inflammatory effects of Oseltamivir [53].

No animal study has been conducted of the infection model with mild and non-lethal doses of the Influenza virus for Zanamivir, Laninamivir, or Peramivir. Only animal studies of the infection model using lethal doses of the Influenza virus are available.

\section{In vitro findings showing inhibitory effects on immune cells}

Peripheral T-lymphocytes from healthy adult whole blood were incubated with antigen presenting cells (APCs) pre-sensitized with Influenza viruses and were tested for their proliferation ability with and without Oseltamivir carboxylate. Proliferation of the Tlymphocytes was suppressed by $15 \%$ and $20 \%$ when incubated with 1 $\mu \mathrm{M}$ and $10 \mu \mathrm{M}$ of Oseltamivir carboxylate, respectively, compared with the control. Concentration of Oseltamivir carboxylate (OC) of $1 \mu \mathrm{M}$ is compatible with the human clinical concentration of OC $[29,30]$. The pharmaceuticals and medical devices agency (PMDA) and the summary basis of approval (SBA) did not refer to any published paper for these findings $[29,30]$. No published papers with these data could be found.

\section{Mechanism for Delayed Reactions}

\section{Psychiatric and other nervous symptoms}

Psychiatric and nervous symptoms that occur in the very early phase of treatment such as acute behavioural change and respiratory depression leading to death may be due to the effects of unmetabolized Oseltamivir phosphate (OP) on the central nervous system (CNS). If OP has affinity to NMDA receptors $[31,54,55]$ and is used for an extended period of time, it may induce schizophrenic reactions in humans, as shown in the prophylaxis RCTs of Oseltamivir $[9,43]$, by a mechanism similar to that of the sudden onset type reactions.

The symptoms that occur in the late phase of treatment with prolonged duration, such as psychosis, confusion, and aggression, and are frequently observed in the prophylaxis trials (shown in section 3.2.6) may also be due to the effects of Oseltamivir carboxylate (OC) on CNS. Pain in the limbs [9] may also be induced by both mechanisms.

Izumi et al reported that systemic injection of Oseltamivir (50 $\mathrm{mg} / \mathrm{kg}$ i.p.) significantly altered the duration of loss of lightning reflex following ethanol injection in rats. Ethanol injection in the presence of Oseltamivir also resulted in enhanced hypothermia [56]. Izumi et al also reported that combination of Oseltamivir with other neurostimulants may alter synaptic plasticity and this may contribute to behavioural changes associated with the drug [57].

As described in section 3.2.4, QT prolongation is closely related to the plasma concentration of Oseltamivir carboxylate.

Taking these into account, it may be possible that Oseltamivir carboxylate directly alters the cell excitability of both neurons and heart muscles, although it is not known whether the alteration is derived from inhibition of the host's endogenous neuraminidase or from other mechanisms, including effects on other receptors or enzymes. Among receptors or enzymes that were tested by Lindeman et al. [58], those that showed apparent dose-related increase are listed in Table 2.

\begin{tabular}{|c|c|c|c|c|}
\hline \multirow[t]{3}{*}{$\begin{array}{l}\text { Target of molecule (receptor etc.)/ } \\
\text { method of assay }\end{array}$} & \multirow{2}{*}{\multicolumn{2}{|c|}{$\begin{array}{c}\begin{array}{c}\text { Inhibition }(\% \\
\text { control) }\end{array} \\
\begin{array}{c}\text { Concentration of } \\
\text { OT }\end{array}\end{array}$}} & \multirow{2}{*}{\multicolumn{2}{|c|}{$\begin{array}{c}\begin{array}{c}\text { Inhibition (\% } \\
\text { control) }\end{array} \\
\begin{array}{c}\text { Concentration of } \\
\text { OC }\end{array}\end{array}$}} \\
\hline & & & & \\
\hline & $3 \mu \mathrm{M}$ & $30 \mu \mathrm{M}$ & $3 \mu \mathrm{M}$ & $30 \mu \mathrm{M}$ \\
\hline Adenosine A receptor & 8 & 13 & 15 & 27 \\
\hline L-type Ca2 ${ }^{+}$channel (diltiazem site) & 14 & 41 & 10 & 9 \\
\hline $\mathrm{Na}^{+}$channel (site 2) & 11 & 38 & n.i.d & 9 \\
\hline NMDA-type glutamate receptor (PCP) & 14 & 23 & 21 & 12 \\
\hline AMPA-type glutamate receptor & 4 & 17 & n.i.d & n.i.d \\
\hline $\begin{array}{l}\text { Glycine-site on NMDAR (strychnine- } \\
\text { insens.) }\end{array}$ & n.i.d & n.i.d & 1 & 24 \\
\hline Kainate-type glutamate receptor & n.i.d & 14 & 10 & 10 \\
\hline Muscarinic receptor M4 & n.i.d & n.i.d & 1 & 10 \\
\hline Neuropeptide Y receptor $\mathrm{Y} 1$ & 8 & 6 & n.i.d & 11 \\
\hline Nociceptin receptor & 9 & 8 & 8 & 16 \\
\hline Norepinephrine transporter & n.i.d & n.i.d & 5 & 11 \\
\hline $\begin{array}{l}\begin{array}{c}\text { Nicotinic acetylchol receptor ( } \alpha 4 \beta 3, \text { BGTX } \\
\text { insens.) }\end{array}\end{array}$ & 5 & n.i.d & n.i.d & 11 \\
\hline hERG potassium channel (Kv11.1) & n.i.d & 17 & 8 & 16 \\
\hline Sigma receptor (non-selective) & 9 & 34 & n.i.d & 3 \\
\hline GABA transporter & 15 & 15 & n.i.d & 17 \\
\hline Serotonin 5-HT2A receptor & 4 & 13 & 15 & n.i.d \\
\hline Serotonin 5 -HT4e receptor & n.i.d & 13 & n.i.d & n.i.d \\
\hline Chemokine receptor 1 (CD191) & 3 & 2 & 2 & 8 \\
\hline Chemokine receptor 3 (CD193) & 10 & 18 & 15 & 12 \\
\hline CXC chemokine receptor 2 (IL-8B) & 1 & 2 & 11 & 16 \\
\hline
\end{tabular}

Table 2: Activity of OP and OC against molecular targets of high relevance for mood, cognition and behavior in binding or functional assay (data are extracted from ref [58]).

Muraki et al. [55] demonstrated that Oseltamivir, but not Oseltamivir carboxylate, directly blocks human neuronal nAChRs. Hiasa et al. [59] found that Oseltamivir, but not Oseltamivir carboxylate, competitively and selectively inhibited human MAO-A. They estimated the Ki value to be 25 to $28 \mu \mathrm{M}$, and IC50 was shown to be between 50 to $100 \mu \mathrm{M}$ in their paper, while Lindeman et al reported that both Oseltamivir and Oseltamivir carboxylate lacked clinically relevant pharmacological activities on a panel of 155 other molecular targets, including MAO-A. Differing results between the study by Lindeman et al and those by Muraki et al or Hiasa et al. may be derived from the different assay methods used.

Accordingly, it is possible that there exist target receptors or enzymes that Oseltamivir carboxylate specifically acts on. 


\section{Attenuated antibody production, immune suppression, and re-infection}

Attenuated antibody production [32-34] may be derived from the same mechanisms as those for reduction of cytokine induction related to inhibition of the host's neuraminidase due to decreased expression of GM1 ganglioside in various immune cells [44-47]. These findings are supported by the fact that both Oseltamivir and Zanamivir reduce antibody production at certain doses both in humans and other animals.

Marois et al [47] demonstrated that Influenza specific CD8+ effector $\mathrm{T}$ cell recruitment was reduced up to $81 \%$ in the lungs of mice treated with Oseltamivir (5 or $50 \mathrm{mg} / \mathrm{kg}$ twice daily; EC50 $49 \mathrm{nM}$ in vitro) compared to saline controls. They also showed that Oseltamivir administration significantly decreased the pools of tissue-resident and circulating effector memory (93.7\%) and central memory CD8 ${ }^{+}$Tcells (45\%) compared to saline controls. During heterologous secondary infection, a decreased memory $\mathrm{CD}^{+} \mathrm{T}$ cell pool combined with reduced generation of secondary Influenza-specific effectors in the lymph nodes resulted in 10 -fold decreased $\mathrm{CD}^{+}{ }^{+}$Tcell recall responses, which increased mouse morbidity and delayed viral clearance. Furthermore, they reported that antiviral administration led to a significant 5.7-fold decreased production of functional anti-Influenza antibodies. They summarized that Oseltamivir treatment affects the kinetics, magnitude, and nature of innate, adaptive, and memory immune responses during PR8 (H1N1) Influenza infection in the mouse model. They suggested that administration of Oseltamivir in infected individuals might reduce the generation of protective immunity against reinfection and, thus, lead to the development of disease [47].

The evidence of re-infection in the subsequent season [33] or within the same season [38,39] supports the adverse effect of both neuraminidase inhibitors on the immune system.

These findings are also consistent with the evidence on the mechanism of action of Oseltamivir from animal models [49,53], a randomized controlled experimental human Influenza study [25], and in vitro findings showing inhibitory effects on immune cells [29].

\section{Other adverse reactions: renal, metabolic, cardiac, prolonged psychiatric and bleeding disorders, pneumonia, etc.}

In mammalian cells, 4 types of sialidase (neuraminidase) have been identified. They are classified according to their major intracellular localization as intralysosomal sialidase (NEU1), cytosolic sialidase (NEU2), plasma membrane-associated sialidases (NEU3), and mitochondrial sialidase (NEU4) [60,61].

Hepatic NEU3 may be associated with sensitivity to insulin and glucose tolerance through modification of ganglioside composition and peroxisome proliferator-activated receptor gamma signaling [62].

Clinical administration of Oseltamivir at doses that result in inhibition of Influenza may also have an additional effect by decreasing GM1 levels in various cells, including immune cells [44-47], nociceptive neurons [48], insulin or peroxisome proliferator-activated receptor gamma signaling [62], and possibly other important cells in the kidney, liver, heart, or central nervous system.

The evidence from these reports suggests that reduction of human endogenous sialidase (neuraminidase) activity by Oseltamivir carboxylate may cause delayed type adverse reactions to neuraminidase inhibitors. These include not only inhibition of antibody and pro-inflammatory cytokine induction, but also prolonged neuropsychiatric reactions, hyperglycemia, renal and hepatic impairment, pneumonia, and exacerbation of infection, such as re-infection of Influenza, gastrointestinal tract hemorrhage, and others.

\section{Difference between Oseltamivir and Other Neuraminidase Inhibitors in Delayed Reactions}

Sufficient plasma concentration of Oseltamivir carboxylate, a metabolite of orally administered Oseltamivir phosphate, acts on the host's endogenous neuraminidase to reduce (or suppress) the immune response even at the dose of $20 \mathrm{mg}$ b.i.d. for 5 days [25]. However, bioavailability of inhaled Zanamivir is $11 \%$, estimated using the data of area under the curve (AUC) from inhalation and intravenous administration over 30 minutes while peak concentration $\left(\mathrm{C}_{\max }\right)$ was $3.1 \%$ of that of intravenously administered Zanamivir [63]. According to the data from the summary basis of approval of Zanamivir in Japan [64], bioavailability of inhaled Zanamivir is calculated to be approximately $9 \%$ to $72 \%$ (geometric mean=25\%). The low bioavailability of Zanamivir may be the major reason why reduction of antibody rise was not observed in the systematic review of Zanamivir [9].

However, if Zanamivir is administered at a high dose or for an extended period, or if the patient is very susceptible, inhaled Zanamivir might reach a concentration high enough to reduce the immune response. In fact, $20 \mathrm{mg} /$ day of Zanamivir for 14 days showed significant reduction of antibody titres to the H1N1 compared with a placebo [36]. Levels of various pro-inflammatory cytokines and chemokines were almost completely suppressed in the viral challenge RCT using a very high dose $(600 \mathrm{mg})$ of intravenous Zanamivir before inoculation of Influenza virus in human adults [37].

Although no report on the suppression of cytokine induction by laninamivir was found in either published papers or the summary basis of approval [65], several reports indicated that pro-inflammatory cytokine induction was suppressed by peramivir administration in the animal models of lethal Influenza virus infection [49-51]. Prolonged median survival was reported in these animal infection models with almost $100 \%$ mortality. However, prolonged survival was not stated in the ferret infection model of Laninamivir [65]. Moreover, viral titres in the nasal wash were higher at 72 hours after inoculation of Influenza virus in ferrets administered with inhaled laninamivir 4 hours after inoculation compared with vehicle control, while they were lower at 24 hours and 48 hours after inoculation [65].

The AUC at the maximum animal dose of Zanamivir $(191 \mu \mathrm{gh} / \mathrm{ml}$ after intravenous $90 \mathrm{mg} / \mathrm{kg}$ ) [64] in rats was only 2.2 times higher than that of healthy male adults [63] $(86.6 \mu \mathrm{gh} / \mathrm{ml}$ after intravenous administration of $600 \mathrm{mg}$ over 30 minutes). The maximum dose of inhaled Laninamivir in the toxicity tests was 7.4 times higher than the usual clinical dose based on the AUC [65]. Hence, it may be hard to detect renal toxicity of Zanamivir and Laninamivir using existing animal toxicity studies.

According to the Japanese summary basis of approval [66], the kidney is a toxic target organ of Peramivir. NOAEL of peramivir for renal toxicity in rabbits was $100 \mathrm{mg} / \mathrm{kg}$, which is $32 \mathrm{mg} / \mathrm{kg}$ in HED (conversion factor 3.1) or 2.7 times higher than the maximum clinical dose of Peramivir $(600 \mathrm{mg} / 50 \mathrm{~kg})$. The safety margin for renal toxicity was estimated at 3.0 and 2.1 based on the AUC in single-dose and 
multiple-dose (7 days) oral rabbit toxicity studies [66]. It is very important to use sensitive animals to detect early signs of toxicity [67]. For example, canaries were used by miners to detect the increased level of carbon monoxide [68].

Inhibitory effects on antibody production have been reported for Oseltamivir and Zanamivir. Inhibitory effects of cytokines or chemokines have been reported for Oseltamivir, Zanamivir, and Peramivir. Toxicities in kidneys have been reported for Oseltamivir and Peramivir. On the other hand, toxicities affecting other cells and cell functions, including effects on ganglioside GM1, have not been investigated, except for Oseltamivir.

Based on these findings, it can be said that neuraminidase inhibitors in general may act as inhibitors of the host's endogenous neuraminidase and thereby induce various delayed type reactions as shown in the case of Oseltamivir.

\section{Summary and Conclusion}

Neuraminidase inhibitors (NIs) are generally believed to relieve Influenza symptoms by inhibiting the neuraminidase of the Influenza virus. However, a clinically compatible dose of Oseltamivir relieves symptoms in mice infected with RSV that lacks neuraminidase. The underlying mechanisms of these phenomena are explained by the inhibition of the host's endogenous neuraminidase, leading to reduction of GM1 ganglioside in immune cells. This may cause subsequent reduction of pro-inflammatory cytokines such as IL-6, interferon, and TNF- $\alpha$. These mechanisms may be closely related to symptom relief without significant reduction of viral load. They also cause attenuated antibody production of secretory IgA antibodies and plasma HI antibodies of Influenza, and may be the cause of reinfection of Influenza within the same or the subsequent seasons.

Mechanisms for delayed onset reactions with prolonged course, such as psychosis and other psychiatric reactions, and renal, metabolic (hyperglycemia or diabetes), and cardiac reactions (QT prolongation), may be related to inhibition of the host's endogenous neuraminidase.

\section{Conflict of Interest}

Rokuro Hama was a co-recipient of a UK National Institute for Health Research grant (HTA 10/80/01, Update and amalgamation of two Cochrane reviews: neuraminidase inhibitors for preventing and treating Influenza in healthy adults and children (www.nets.nihr.ac.uk/ projects/hta/108001). RH wrote two books published in 2008 about the harm of Oseltamivir and antipyretics. He provided scientific opinions and expert testimony on 14 adverse reaction cases related to Oseltamivir for the applications by their families for adverse reaction relief by PMDA (Pharmaceuticals and Medical Devices Agency) and in the lawsuits for revocation of the PMDA's decision concerning with these reactions. Most of the cases were reported in reference [6].

\section{References}

1. CDC (2009) Interim Guidance on Antiviral Recommendations for Patients with Novel Influenza A (H1N1) Virus Infection and Their Close Contacts. Center for Disease control and Prevention.

2. CDC (2015) Influenza antiviral medications: summary for clinicians. Center for Disease control and Prevention.

3. World Health Organization (2015) WHO Model List of Essential Medicines.
4. Maxwell SRJ (2007) Tamiflu and neuropsychiatric disturbance in adolescents. BMJ 334: 1232-1233.

5. Hama R (2005) Discussion of the causal relationship between Oseltamivir phosphate (Tamiflu), and sudden death and death from abnormal behavior. Japanese Society for Pediatric Infectious Diseases in Tsu, Mie Prefecture.

6. Hama R (2008) Fatal neuropsychiatric adverse reactions to Oseltamivir: case series and overview of causal relationships. International Journal of Risk and Safety in Medicine; 20: 5-36.

7. Hama R (2007) Oseltamivir's adverse reactions: Fifty sudden deaths may be related to central suppression. BMJ. 335: 59.

8. Hama R. Jones M, Hayashi K, Sakaguchi K (2011) Oseltamivir and early deterioration leading to death. Int J Risk Safety Med 23: 201-215.

9. Jefferson T, Jones MA, Doshi P, Del Mar CB, Hama R, et al. (2014) Neuraminidase inhibitors for preventing and treating Influenza in healthy adults and children. Cochrane Database Syst Rev 4: CD008965.

10. Jefferson T, Jones MA, Doshi P, Del Mar CB, Heneghan CJ, et al (2012) Neuraminidase inhibitors for preventing and treating Influenza in healthy adults and children. Cochrane Database Syst Rev 1: CD008965.

11. Kohno S, Yen MY, Cheong HJ, Hirotsu N, Ishida T, et al. (2011)Clinical Study Group (2011) Phase III randomized, double-blind study comparing single-dose intravenous peramivir with oral Oseltamivir in patients with seasonal Influenza virus infection. Antimicrob Agents Chemother 55: 5267-5276.

12. Sugaya N, Ohashi Y (2010) Long-acting neuraminidase inhibitor laninamivir octanoate (CS-8958) versus Oseltamivir as treatment for children with Influenza virus infection. Antimicrobial Agents and Chemotherapy 54: 2575-2582.

13. Hayden F (2009) Developing new antiviral agents for Influenza treatment: what does the future hold?. Clinical Infectious Diseases 48: S3S1.

14. Roche. Media release (2006) Roche donates a further 2 million treatment courses of antiviral Tamiflu to the WHO for regional stockpiling.

15. Jack A (2009) Roche steps up production of Tamiflu after virus scare.

16. FDA Pediatric Advisory Committee Meeting (2007) Relenza (Zanamivir) Inhalation Powder NDA 21-036. Glaxosmithkline.

17. http://www.fda.gov/ohrms/dockets/ac/07/briefing/ 2007-4325b_02_16_Sponsor\%20Background\%20Package\%20GSK.pdf

18. http://www.mhlw.go.jp/file/05-Shingikai-11121000Iyakushokuhinkyoku-Soumuka/0000063406.pdf

19. Bhatia A, Kast RE (2007) How Influenza's neuraminidase promotes virulence and creates localized lung mucosa immunodeficiency. Cell Mol Biol Lett 12: 111-119.

20. Matrosovich MN, Matrosovich TY, Gray T, Roberts NA, Klenk H (2004) Neuraminidase is important for the initiation of Influenza virus infection in human airway epithelium. J Virol 78: 12665-12667.

21. Moscona A (2005) Neuraminidase inhibitors for Influenza. N Engl J Med 353: 1363-1373.

22. Ohuchi M, Asaoka N, Sakai T, Ohuchi R (2006) Roles of neuraminidase in the initial stage of Influenza virus infection. Microbes and Infection 8: 1287-1293.

23. Liu C, Eichelberger MC, Compans RW, Air GM (1995) Influenza type A virus neuraminidase does not play a role in viral entry, replication, assembly, or budding. J Virol. 69:1099-1106.

24. http://www.accessdata.fda.gov/drugsatfda_docs/label/ 2011/021087s057lbl.pdf

25. Hayden FG, Treanor JJ, Fritz RS, Lobo M, Betts RF, et al. (1999) Use of the oral neuraminidase inhibitor Oseltamivir in experimental human Influenza: randomized controlled trials for prevention and treatment. JAMA 282: 1240-1246.

26. Morimoto K, Nakakariya M, Shirasaka Y, Kakinuma C, Ogihara T, et al. (2008) Oseltamivir (Tamiflu) efflux transport at the blood-brain barrier via P-glycoprotein. Drug Metab Dispos 36: 6-9. 
27. Ose A, Kusuhara H, Yamatsugu K, Kanai M, Sugiyama Y (2008) Pglycoprotein restricts the penetration of Oseltamivir across the bloodbrain barrier.Drug Metab Dispos 36: 427-434.

28. http://www.mhlw.go.jp/shingi/2007/12/s1225-7.html

29. http://www.pmda.go.jp/drugs/2000/g001202/index.html

30. http://www.pmda.go.jp/drugs/2000/g001202/70repo01.pdf

31. Hama R (2015) The mechanisms of adverse reactions to Oseltamivir: Part I. Sudden-onset type reactions. J clinical toxicology 5: 1-10.

32. Sawabuchi T, Suzuki S, Iwase K, Ito C, Mizuno D, Kido H, et al. (2009) Boost of mucosal secretory immunoglobulin A response by clarithromycin in paediatric Influenza. Respirology 14: 1173-1179.

33. Shinahara W, Takahashi E, Sawabuchi T, Arai M, Kido H, et al. (2013) Immunomodulator clarithromycin enhances mucosal and systemicimmune responses and reduces re-infection rate in pediatric patients with Influenza treated with antiviral neuraminidase inhibitors: a retrospective analysis.PLoS One 8: e70060.

34. Takahashi E, Kataoka K, Fujii K, Chida J, Kido H, et al. (2010) Attenuation of inducible respiratory immune responses by Oseltamivir treatment in mice infected with Influenza A virus. Microbes Infect 12: 778-783.

35. Takahashi E, Kataoka K, Indalao IL, Konoha K, Kido H et al (2012) Oral clarithromycin enhances airway immunoglobulin A (IgA) immunity through induction of IgA class switching recombination and B-cellactivating factor of the tumor necrosis factor family molecule on mucosal dendritic cells in mice infected with Influenza A virus. J Virol 86: 10924-10934.

36. Cox RJ, Mykkeltvedt E, Sjursen H, Haaheim LR (2001) The effect of Zanamivir treatment on the early immune response to Influenza vaccination. Vaccine 19: 4743-4749.

37. Fritz RS, Hayden FG, Calfee DP, Cass LM, Straus SE, et al. (1999) Nasal cytokine and chemokine responses in experimental Influenza A virus infection: results of a placebo-controlled trial of intravenous Zanamivir treatment. J Infect Dis 180: 586-593.

38. Kopel E, Mandelboim M, Amitai Z, Grotto I, Hindiyeh M, et al. (2010) Possible recurrent pandemic (H1N1) 2009 infection, Israel. Emerg Infect Dis 16: 1321-1322.

39. Perez CM, Ferres M, Labarca JA (2010 Pandemic (H1N1) 2009 reinfection, Chile. Emerg Infect Dis 16: 156-157.

40. U.S. Department of Health and Human Services (2015) Guidance for Industry-Estimating the Maximum Safe Starting Dose in Initial Clinical Trials for Therapeutics in Adult Healthy Volunteers, Food and Drug Administration, Center for Drug Evaluation and Research (CDER), USA.

41. Brewster M (1999) Cardiovascular and respiratory evaluation in the anaesthetized dog following intravenous administrations.

42. Hama R, Jones M, Jefferson $\mathrm{T}$ (2015) Concerns about the randomization in the prophylaxis trials of Oseltamivir. BMJ 348: g2545.

43. The Dryad Digital Repository (2014) Neuraminidase inhibitors for preventing and treating influenza in healthy adults and children.

44. Moore ML, Chi MH, Zhou W, Goleniewska K, Peebles RS Jr, et al. (2007) Cutting edge: Oseltamivir decreases T cell GM1 expression and inhibits clearance of respiratory syncytial virus: potential role of endogenous sialidase in antiviral immunity. Journal of Immunology 178: 2651-2654.

45. Stamatos NM, Carubelli I, van de Vlekkert D, Bonten EJ, Papini N et al (2010) LPS-induced cytokine production in human dendritic cells is regulated by sialidase activity. Leukoc Biol 88: 1227-1239.

46. Feng C, Zhang L, Almulki L, Faez S, Cross AS, et al. (2011) Endogenous PMN sialidase activity exposes activation epitope on CD11b/CD18 which enhances its binding interaction with ICAM-1. J Leukoc Biol 90: 313-321.

47. Marois I, Cloutier A, Garneau É, Lesur O, Richter MV (2015) The administration of Oseltamivir results in reduced effector andmemory CD8+ T cell responses to Influenza and affects protective immunity. FASEB J 29: 973-987.

48. Crain SM, Shen KF (2004) Neuraminidase inhibitor, Oseltamivir blocks GM1 ganglioside-regulated excitatory opioid receptor-mediated hyperalgesia, enhances opioid analgesia and attenuates tolerance in mice. Brain Research 995: 260-266.

49. Mendel DB, Tai CY, Escarpe PA, Li W, Sidwell RW, et al. (1998) Oral administration of a prodrug of the Influenza virus neuraminidase inhibitor GS 4071 protects mice and ferrets against Influenza infection. Antimicrobial Agents and Chemotherapy 42:640-646.

50. Onishi M, Kitano M, Taniguchi K, Homma T, Sato A, et al. (2015) Intravenous peramivir inhibits viral replication, and leads to bacterial clearance and prevention of mortality during murine bacterial coinfection caused by Influenza A(H1N1)pdm09 virus and Streptococcus pneumoniae. Antiviral Res 117: 52-59.

51. Tanaka A, Nakamura S, Seki M, Iwanaga N, Kajihara T, et al. (2015) The effect of intravenous peramivir, compared with oral Oseltamivir, on the outcome of post-Influenza pneumococcal pneumonia in mice.Antivir Ther 20:11-19.

52. Kitano M, Kodama M, Itoh Y, Kanazu T, Sato A (2013) Efficacy of repeated intravenous injection of peramivir against Influenza A (H1N1) 2009 virus infection in immunosuppressed mice. Antimicrob Agents Chemother 57:2286-2294.

53. Wong ZX, Jones JE, Anderson GP, Gualano RC (2011) Oseltamivir treatment of mice before or after mild Influenza infection reduced cellular and cytokine inflammation in the lung. Influenza Other Respiratory Viruses 5: 343-350.

54. Hama R (2009) Pharmacological toxicological clinical and epidemiological evidences of neuropsychiatric adverse reactions to Oseltamivir and misleading analysis due to serious miscalculation in Japanese MHLW's 2006/07 epidemiological study report. Jap J Clin Pharmacol Therap 40: 13S-14S.

55. Muraki K, Hatano N, Suzuki H, Muraki Y, Iwajima Y, Ono H, et al. (2014) Oseltamivir Blocks Human Neuronal Nicotinic Acetylcholine ReceptorMediated Currents. Basic Clin Pharmacol Toxicol 116: 87-95.

56. Izumi Y, Tokuda K, O'dell KA, Zorumski CF, Narahashi T (2007) Neuroexcitatory actions of Tamiflu and its carboxylate metabolite. Neurosci Lett 426: 54-58.

57. Izumi Y, Tokuda K, O'Dell K, Zorumski C, Narahashi T (2008) Synaptic and behavioral interactions of Oseltamivir (Tamiflu) with neurostimulants. Hum Exp Toxicol 27: 911-917.

58. Lindemann L, Jacobsen H, Schuhbauer D, Knoflach F, Gatti S, et al. (2010) In vitro pharmacological selectivity profile of Oseltamivir prodrug (Tamiflu) and active metabolite. Eur J Pharmacol 628: 6-10.

59. Hiasa M, Isoda Y, Kishimoto Y, Saitoh K, Kuzuhara T, et al. (2013) Inhibition of MAO-A and stimulation of behavioural activities in mice by the inactive prodrug form of the anti-Influenza agent Oseltamivir. $\mathrm{Br} \mathrm{J}$ Pharmacol 169: 115-129.

60. Yamaguchi K, Hata K, Koseki K, Shiozaki K, Miyagi T, et al. (2005) Evidence for mitochondrial localization of a novel human sialidase (NEU4). Biochem J 390(Pt 1): 85-93.

61. Yamanami H, Shiozaki K, Wada T, Yamaguchi K, Miyagi T, et al. (2007) Down-regulation of sialidase NEU4 may contribute to invasive properties of human colon cancers. Cancer Sci 98: 299-307.

62. Yoshizumi S, Suzuki S, Hirai M, Hinokio Y, Miyagi T, et al. (2007) Increased hepatic expression of ganglioside-specific sialidase, NEU3, improves insulin sensitivity and glucose tolerance in mice. Metabolism 56: 420-429.

63. Shelton MJ, Lovern M, Ng-Cashin J, Jones L, Gould E et al. (2011) Zanamivir pharmacokinetics and pulmonary penetration into epithelial lining fluid following intravenous or oral inhaled administration to healthy adult subjects. Antimicrob Agents Chemother 55: 5178-5184.

64. http://www.pmda.go.jp/drugs/1999/g991201/index.html

65. http://www.pmda.go.jp/drugs/2010/P201000050/index.html

66. http://www.pmda.go.jp/drugs/2010/P201000001/index.html

67. Chengelis CP, Gad SC (1992) Animal Model in Toxicology. Marcel Dekker. Inc. New York, USA. 
Citation: Hama R (2015) The Mechanisms of Adverse Reactions to Oseltamivir: Part II. Delayed Type Reactions. Clin Microbiol 4: 224. doi: 10.4172/2327-5073.1000224

68. Burrell GA (1912) The use of Mice and Birds for detecting Carbon Monoxide After Mine fire and Explosion. US Dept. of the Interior, Bureau of Mines, USA. 